\title{
A GONVOLUTION SEMIGROUP OF MODULAR FUNCTIONS
}

\author{
Y.-F. LIN \\ (Received 8 December 1965)
}

\section{Introduction}

Let $S$ be a compact topological semigroup, and let $S$ be the collection of all normalized non-negative Borel measures on $S$. It is well-known that $\tilde{S}$, under convolution and the topology induced by the weak-star topology on the dual of the Banach space $C(S)$ of all complex valued continuous functions on $S$, forms a compact topological semigroup which is known as the convolution semigroup of measures (see for instance, Glicksberg [3], Collins [1], Schwarz [5] and the author [4]). Professor A. D. Wallace asked if the process of forming the convolution semigroup of measures might be generalized to a more general class of set functions, the so-called "modular functions." The purpose of the present note is to settle this question in the affirmative under a slight restriction. Before we are able to state the Wallace problem precisely, some preliminaries are necessary.

\section{Preliminaries}

Let $S$ in this note be always a compact topological semigroup, and let $\mathscr{F}$ be the family of all closed subsets of $S$. By a modular function $m$ on $S$ is meant a real-valued set function $\boldsymbol{m}$ defined on $\mathscr{F}$ such that

$$
m(A \cup B)+m(A \cap B)=m(A)+m(B),
$$

for any $A$ and $B$ in $\mathscr{F}$. A modular function is said to be normalized if $m(S)=1$ and $m(\square)=0$, where $\square$ denotes the empty set.

DEFInition 1. A modular function $m$ on $S$ is regular if and only if, for any $F$ in $\mathscr{F}$ and any $\varepsilon>0$, there is an open subset $V$ of $S$ containing $F$ such that $0 \leqq m(E)-m(F)<\varepsilon$ for any $E$ in $\mathscr{F}$ such that $F \subset E \subset V^{-}$, where - denotes the topological closure operator.

Let us write $S \#$ for the family of all normalized regular modular functions on $S$. We are now in position to restate Wallace's problem more clearly: is it possible to define a "convolution" and a topology for $S \#$ in such a way that $S \#$ becomes a compact topological semigroup? 
We are able to answer this for the "isotonic" modular functions. A modular function is isotone if and only if, $A \supset B$ in $\mathscr{F}$ implies $m(A) \geqq m(B)$.

Let us agree, henceforth, that $S \#$ is the collection of all normalized regular isotonic modular functions on $S$. An example of such a function is a normalized regular Borel measure on $S$ restricted to $\mathscr{F}$. It is rather peculiar that the modular functions obtained in this way turn out to be all of $S \#$, and furthermore there is a unique extension of an element of $S \not \#$ to an element of $\widetilde{S}$.

We need some additional symbolism. Let $\mathscr{B}$ denote the family of all Borel sets in $S$, and let $\mathscr{V}$ be the collection of all open sets in $S$. We write

$$
m_{0}(V)=1-m(S \mid V),
$$

for each $m$ in $S \#$ and for all $V$ in $\mathscr{V}$. We then define a transformation ${ }^{t}$ on $S \#$ by

$$
m^{t}(B)=\inf \left\{m_{0}(V): B \subset V \varepsilon \mathscr{V}\right\}
$$

for each $m$ in $S \not \#$ and for all Borel sets $B$ in $\mathscr{B}$.

\section{A convolution semigroup of modular functions}

TheOREM A. The transformation ${ }^{t}$ takes $S \#$ in one-to-one fashion onto $\widetilde{S}$. Indeed, we have the following relations:

$$
m^{t} \mid \mathscr{F}=m \text { and }(\mu \mid \mathscr{F})^{t}=\mu
$$

for every $m$ in $S \#$ and for every $\mu$ in $\bar{S}$.

We divide the proof of this theorem into the following steps.

LEMma 1. If $U, V$ are in $\mathscr{V}$ and if $F$ is in $\mathscr{F}$ such that $U \subset F \subset V$. Then

$$
m_{0}(U) \leqq m(F) \leqq m_{0}(V)
$$

for any $m$ in $S \#$.

Proof: This is straightforward from (1).

LeMma 2. The set function $m_{0}$ is normalized, isotonic, countably additive on $\mathscr{V}$ for each $m$ in $S \#$.

Proof. It is clear that $m_{0}(\square)=0, m_{0}(S)=1$ and that

$$
U=U^{0} \supset V^{0}=V
$$

implies $m_{0}(U) \geqq m_{0}(V)$. Furthermore, $m_{0}$ is finitely additive; for if $U$ and $V$ are any disjoint open sets, then 


$$
\begin{aligned}
m_{0}(U \cup V) & =1-m((S \mid U) \cap(S \mid V)) \\
& =1-[m(S \mid U)+m(S \mid V)-m((S \mid U) \cup(S \mid V))] \\
& =[1-m(S \mid U)]+[1-m(S \mid V)] \\
& =m_{0}(U)+m_{0}(V)
\end{aligned}
$$

for, $S=(S \mid U) \cup(S \mid V)$ and $m$ is a normalized modular function.

Now we show that for any sequence $\left\{V_{i}: i \geqq 1\right\}$ of open sets $V_{i}$,

$$
\sum_{i \geqq 1} m_{0}\left(V_{i}\right) \geqq m_{0}\left(\bigcup_{i \geqq 1} V_{i}\right)
$$

For any $\varepsilon>0$, since $S \backslash\left(\bigcup_{i \geq 1} V_{i}\right)=\bigcap_{i \geqq 1}\left(S \mid V_{i}\right) \in \mathscr{F}$ and by regularity of $m$, there is an open set $W$ containing $\bigcap_{i \geqq 1}\left(S \mid V_{i}\right)$ such that

$$
m\left(\bigcap_{i \geqq 1}\left(S \mid V_{i}\right)\right)+\varepsilon \geqq m\left(W^{-}\right) .
$$

Compactness of $S$, then, yields a positive integer $n$ such that

$$
W \supset \bigcap_{n \geq i \geq 1}\left(S \mid V_{i}\right)
$$

It follows then from (1), (3), (4), (6), and (7) that

and hence

$$
\sum_{n \geqq i \geqq 1} m_{0}\left(V_{i}\right)+\varepsilon \geqq m_{0}\left(\bigcup_{i \geqq 1} V_{i}\right)
$$

$$
\sum_{i \geqq 1} m_{0}\left(V_{i}\right)+\varepsilon \geqq m_{0}\left(\bigcup_{i \geqq 1} V_{i}\right) .
$$

Since $\varepsilon$ was arbitrary, (5) is thus proved.

Finally, with an additional assumption that $\left\{V_{i}: i \geqq 1\right\}$ is disjoint, we have to show that

$$
\sum_{i \geq 1} m_{0}\left(V_{i}\right) \leqq m_{0}\left(\bigcup_{i \geqq 1} V_{i}\right)
$$

From (3) and (4), we have

$$
\sum_{n \geq i \geqq 1} m_{0}\left(V_{i}\right)=m_{0}\left(\bigcup_{n \geqq i \geqq 1} V_{i}\right) \leqq m_{0}\left(\bigcup_{i \geqq 1} V_{i}\right)
$$

for every positive integer $n$. Therefore (8) holds, and hence the lemma is proved.

LEMMA 3. If $m \varepsilon S \#$ then $m^{t}$ is a countably additive measure on $\mathscr{B}$.

Proof. From (2) we have $m^{t}(\square)=0$. Let $\left\{B_{i}: i \geqq 1\right\}$ be a sequence of Borel sets such that $\bigcup\left\{B_{i}: i \geqq 1\right\}$ is also a Borel set. Then for any $\varepsilon>0$ and for each positive integer $i$, there is an open set $V_{i} \supset B_{i}$ such that

$$
m_{0}\left(V_{i}\right) \leqq m^{t}\left(B_{i}\right)+\frac{\varepsilon}{2^{i}} .
$$


Therefore, from (4) and (5), we have

and thus,

$$
m^{t}\left(\bigcup_{i \geqq 1} B_{i}\right) \leqq m_{0}\left(\bigcup_{i \geqq 1} V_{i}\right) \leqq \sum_{i \geqq 1} m_{0}\left(V_{i}\right) \leqq \sum_{i \geqq 1} m^{t}\left(B_{i}\right)+\varepsilon,
$$

$$
m^{t}\left(\bigcup_{i \geqq 1} B_{i}\right) \leqq \sum_{i \geqq 1} m^{t}\left(B_{i}\right)
$$

These together with the isotony of $m^{t}$ show that $m^{t}$ is an outer measure on the $\sigma$-algebra $\mathscr{B}$.

Since $m$ is regular, by some standard computations, all sets in $\mathscr{F}$ are $m^{t}$-sets (= outer measurable sets). Now a celebrated theorem of Carathéodory (see for instance $[2, \mathrm{p} .134]$ ), tells us that all Borel sets are $\boldsymbol{m}^{t}$-sets upon which $m^{t}$ is countably additive.

Lemma 4. If $m \in S \#$ then $m^{t}$ is regular.

Proof. This follows from the fact that

$$
\begin{aligned}
m^{t}(F) & =\inf \left\{m_{0}(V): F \subset V \in \mathscr{V}\right\} \\
& =\inf \left\{m^{t}(V): F \subset V \in \mathscr{V}\right\} .
\end{aligned}
$$

Lemma 5. If $m \in S \#$ then $m^{t} \mid \mathscr{F}=m$.

Proof. Since $m$ is regular, for any $F$ in $\mathscr{F}$ and any $\varepsilon>0$ there is an open set $V$ such that

$$
F \subset V \text { and } m\left(V^{-}\right) \leqq m(F)+\varepsilon .
$$

Using (2) and (3) several times we arrive at

$$
m(F) \leqq m^{t}(F) \leqq m_{0}(V) \leqq m\left(V^{-}\right) \leqq m(F)+\varepsilon
$$

and see $m(F)=m^{t}(F)$ for all $F$ in $\mathscr{F}$.

LEMMA 6. If $\mu \in \Phi$ then $(\mu \mid \mathscr{F})^{t}=\mu$.

Proof. It is fairly clear that $\mu \mid \mathscr{F}$ belongs to $S \#$. Let us denote $(\mu \mid \mathscr{F})^{t}$ by $\nu$. Then by Lemma 5 ,

$$
\nu(F)=\mu(F)
$$

for every closed set $F$, and hence

$$
v(V)=\mu(V)
$$

for each open set $V$. We have then, by the regularity of $\mu$ and by (2),

for every Borel set $B$.

$$
\nu(B)=\mu(B)
$$

The proof of Theorem $A$ is now clear from Lemmas 1-6. We are now ready to state our main theorem. 
THEOREM B. Let $S$ be a compact semigroup. Then the set $S \#$ of all normalized regular isotonic modular functions may be introduced a convolution and a topology in such a way that $S \#$ is topologically isomorphic to $\mathbb{S}$.

Proof. We define, by virtue of Theorem $A$, the convolution * on $S \#$ naturally by

$$
m * n=\left(m^{t} \cdot n^{t}\right) \mid \mathscr{F}
$$

for all $m, n$ in $S \#$. Where - in the right hand side means the convolution of measures in the usual sense. Topologize $S \#$ in such a way that a subset $\Sigma$ is open if and only if $\Sigma^{t}=\left\{\sigma^{t}: \sigma \in \Sigma\right\}$ is open in $\widetilde{S}$. Then the mapping $h: S \not \# \rightarrow \mathcal{S}$ defined by $h(m)=m^{t}$ for all $m$ in $S \#$ is an isomorphism as well as a homeomorphism.

The author is grateful to Professor A. D. Wallace for this problem.

\section{Bibliography}

[1] Collins, H. S., 'The kernel of a semigroup of measures', Duke Math. J., 28 (1961) $387-392$.

[2] Dunford, N. and Schwartz, J. T., Linear operators $I$, Interscience Publishers, New York (1958).

[3] Glicksberg, I., 'Convolution semigroups of measures', Pacific J. Math., 9 (1959), 51 -67.

[4] Lin, Y.-F., 'Not necessarily Abelian convolution semigroups of probability measures', Math. Z., 91 (1966), 300-307.

[5] Schwarz, S., 'Probability measures on non-commutative semigroups', Proc. Symp. in General Topology and its Relations to Modern Analysis and Algebra, Prague (1962).

The University of South Florida 